\title{
Seleção e herdabilidade na predição de ganhos genéticos em maracujá-amarelo
}

\author{
Gustavo Menezes Gonçalves ${ }^{(1)}$, Alexandre Pio Viana(1), Francisco Valdevino Bezerra Neto(1), \\ Messias Gonzaga Pereira ${ }^{(1)}$ e Telma Nair Santana Pereira ${ }^{(1)}$
}

\begin{abstract}
(1)Universidade Estadual do Norte Fluminense Darcy Ribeiro, Centro de Ciências e Tecnologias Agropecuárias, Laboratório de Melhoramento Genético Vegetal, Av. Alberto Lamego, 2000, Horto, CEP 28015-620 Campos dos Goytacazes, RJ. E-mail: gustavog@uenf.br, pirapora@uenf.br, fvbn@uenf.br, messias@uenf.br, telmasp@uenf.br
\end{abstract}

\begin{abstract}
Resumo - O objetivo deste trabalho foi avaliar a alternativa de seleção para a população de maracujá-amarelo, estruturada no Delineamento I, para obtenção do melhor ganho genotípico predito. Avaliaram-se seis características produtivas em 113 progênies, nas localidades de Viçosa, MG, e Miracema, RJ, em arranjos de três agrupamentos, com delineamento em blocos ao acaso, com três repetições e três plantas por parcela. Foram empregados, para o cálculo dos ganhos preditos, os índices de seleção de Mulamba \& Mock, Pesek \& Baker, Smith e Hazel e a seleção direta em número de frutos por planta. Foi observada falta de interação entre genótipos e ambiente. As alternativas que demonstraram os maiores ganhos preditos foram a seleção combinada e a seleção entre famílias de machos, com 18,55\% para seleção direta em número de frutos por planta pela seleção combinada. O índice de Smith e Hazel apresentou os menores ganhos preditos. Os índices de Mulamba \& Mock e Pesek \& Baker os maiores ganhos preditos. Os índices aliados às alternativas estudadas têm potencial para a seleção das progênies.
\end{abstract}

Termos para indexação: Passiflora, melhoramento, seleção genotípica, índices de seleção.

\section{Selection and heritability in the prediction of genetic gain in yellow passion fruit}

\begin{abstract}
The objective of this research was to evaluate the alternatives of selection for a population of yellow passion fruit, structured in Design I, for obtaining the best genotypic gain prediction. Six traits were evaluated in 113 progenies at two environments: Viçosa, MG, and Miracema, RJ, in Brazil, grouped in three sets in the randomized blocks design, with three replications and three plants per plot. For gain prediction calculation, the indexes of Mulamba \& Mock, Pesek \& Baker, Smith and Hazel, and the selection based on number of fruits per plants, were used. The absence of interaction genotype versus environment was observed. The alternatives that presented the best genetic gain prediction were the combined selection and selection among male families. Genetic gain prediction, based on combined selection, was $18.55 \%$ for direct selection of fruit number per plant. The indexes of Smith and Hazel presented the lowest genetic gain prediction. The indexes of Mulamba \& Mock and Pesek \& Baker had the highest predicted genetic gain. The indexes allied with alternatives studied have high potential in the selection of the progenies.
\end{abstract}

Index terms: Passiflora, breeding, genotypic selection, selection indexes.

\section{Introdução}

O Brasil é um grande produtor do maracujá-amarelo, com crescente demanda por esta fruta, tanto para o mercado de suco processado, quanto para o da fruta in natura. Entretanto, essa cultura depara-se com fatores limitantes como a baixa produtividade, causada, entre outros fatores, pela falta de genótipos altamente produtivos e pela grande variabilidade existente em pomares comerciais, o que reflete a necessidade do melhoramento genético.

O Delineamento I é utilizado com o objetivo de se estimarem os componentes de variância genética de uma população (Hallauer \& Miranda Filho, 1988; Cruz \& 
Carneiro, 2003). O esquema de famílias deste delineamento, freqüentemente utilizado na cultura do milho (Hallauer \& Miranda Filho, 1988; Eyherabide \& Hallauer, 1991; Furtado, 1996), permite adotar diferentes estratégias de seleção, tais como: seleção entre machos, fêmeas, fêmeas hierarquizadas a machos e seleção combinada (Cruz \& Carneiro, 2003).

De acordo com Cruz et al. (2004), os índices de seleção constituem-se em um caráter adicional, estabelecido pela combinação ótima das várias características analisadas, o que permite que se realize com eficiência a seleção simultânea em vários caracteres.

Cruz et al. (1993) encontraram resultados positivos com a utilização dos índices de Mulamba \& Mock (1978), Elston (1963) e o de Williams (1962), na predição de ganhos em todos os caracteres de seu interesse, quando trabalharam com progênies de irmãos completos de milho, o que não foi possível quando utilizaram a seleção direta e a indireta.

Granate et al. (2002), com o objetivo de comparar os ganhos preditos obtidos na seleção simultânea de caracteres em milho-pipoca, com utilização de vários índices de seleção, concluíram que os ganhos preditos com o índice de Smith (1936) e Hazel (1943) foram superiores aos preditos com outros índices, e manifestaram-se em mais caracteres, além disso, o uso dos índices, de forma geral, permitiu a predição de ganhos simultâneos nas duas principais características, produtividade e capacidade de expansão, mesmo que correlacionadas negativamente.

O objetivo deste trabalho foi o de avaliar as alternativas de seleção para a população de maracujá-amarelo, estruturada no Delineamento I, para obtenção do melhor ganho genotípico predito, obtido por diferentes índices de seleção.

\section{Material e Métodos}

Os experimentos foram instalados nos ambientes de Viçosa, MG $\left(20^{\circ} 45^{\prime} \mathrm{S}\right.$ e $42^{\circ} 53^{\prime} \mathrm{W}$, altitude de $\left.648 \mathrm{~m}\right)$ e em Miracema, RJ ( $21^{\circ} 25^{\prime} \mathrm{S}$ e $42^{\circ} 12^{\prime} \mathrm{W}$, altitude de $\left.137 \mathrm{~m}\right)$, em novembro de 2002, com data final de avaliação em maio de 2004. Nesses ambientes, estudaram-se 113 progênies de maracujá-amarelo, provenientes de cruzamentos que seguiram o Delineamento I, procedimento proposto por Comstock \& Robinson (1948), entre plantas de área de plantio comercial, em Campos dos Goytacazes, RJ, no período de maio a julho de 2002. Essa população de plantas era composta pelas variedades: Maguary, Yellow Master e seleções de São Francisco do Itabapoana, com coeficiente de endogamia $\mathrm{F}=0$. Para isto, uma planta doadora de grãos de pólen foi cruzada com um grupo de cinco plantas receptoras, e formaram progênies de irmãos completos e meiosirmãos, conforme Delineamento I. O delineamento estatístico utilizado foi o de blocos ao acaso, em que cada parcela foi formada por três plantas, com as progênies (tratamentos) arranjadas em três agrupamentos: 1) progênies 1 a 40;2) progênies 41 a 80 ; e 3) progênies 81 a 113 . Os agrupamentos constituíram grupos de tratamentos, e cada grupo foi composto por três repetições.

As avaliações das progênies foram efetuadas durante todo o ciclo de produção da cultura, por meio das seguintes características: número de frutos por planta (NF), avaliado pela contagem do número de frutos provenientes da primeira florada, dividido pelo número de plantas, em cada parcela; peso de frutos (PF), valor médio obtido, em gramas, pela pesagem de amostras de 25 frutos por parcela; comprimento de frutos $(\mathrm{CF})$, valor médio obtido em milímetros, pela medição da dimensão longitudinal de amostras de 25 frutos por parcela; largura de frutos (LF), valor médio obtido em milímetros, pela medição da dimensão transversal de amostras de 25 frutos por parcela; espessura de casca (EC), valor médio obtido em milímetros, pela medição da dimensão da casca na porção mediana dos frutos (cortados transversalmente) em amostras de 25 frutos por parcela; número de dias até a antese (DA), feita por meio da contagem do número de dias transcorridos desde o plantio, em campo, até a abertura da primeira flor na parcela.

Para se verificar a existência ou não de variabilidade na população estudada, realizaram-se análises de variância de cada uma das características avaliadas, pelo modelo genético-estatístico proposto por Hallauer \& Miranda Filho (1988) e Pereira \& Amaral Júnior (2001).

A predição dos ganhos genéticos com a seleção foi realizada considerando-se as alternativas de seleção derivadas do Delineamento I (Furtado, 1996; Cruz \& Carneiro, 2003): seleção baseada na média de machos, seleção baseada na média de fêmeas, seleção baseada na média de fêmeas hierarquizadas a machos e seleção combinada. Para os cálculos dos ganhos genéticos foram utilizados os coeficientes de herdabilidade no sentido restrito, e seus estimadores foram:

1) seleção baseada na média de machos, em que a seleção foi praticada entre os valores médios obtidos para 
cada família de macho, com progênies provenientes de fêmeas polinizadas por um mesmo indivíduo: $\mathrm{h}_{\mathrm{M}}^{2}=\left(\frac{\mathrm{f}+1}{4 \mathrm{f}} \hat{\sigma}_{\mathrm{A}}^{2}\right) / \frac{\mathrm{QM}_{\mathrm{M}}}{\text { ref }} ;$

2) seleção baseada na média de fêmea, em que se praticou a seleção entre famílias de fêmeas, independentemente do macho a que ela pertencia: $\mathrm{h}_{\mathrm{F}}^{2}=\left(\frac{2 \mathrm{mf}-\mathrm{f}-1}{\mathrm{mf}-1} \times \frac{1}{4} \hat{\sigma}_{\mathrm{A}}^{2}\right) / \frac{\mathrm{QM}_{\mathrm{P}}}{\mathrm{re}}$;

3) seleção baseada na média de fêmeas hierarquizadas a machos, em que se praticou a seleção estratificada de famílias de fêmeas, na qual foram selecionadas famílias de fêmeas hierarquizadas a um mesmo macho:

$\mathrm{h}_{\mathrm{F} / \mathrm{M}}^{2}=\left(\frac{1}{4} \hat{\sigma}_{\mathrm{A}}^{2}\right) / \frac{\mathrm{QM}_{\mathrm{F} / \mathrm{M}}}{\mathrm{re}}$,

em que: $h_{M}^{2}, h_{F}^{2}$ e $h_{F / M}^{2}$ são os coeficientes de herdabilidade no sentido restrito, com relação à média de macho, de fêmea e de fêmeas hierarquizadas a macho, respectivamente; r, e, f são o número de repetições, o número de ambientes, e o número de fêmeas, respectivamente; $\hat{\sigma}_{\mathrm{A}}^{2}$ é a estimativa do componente de variância genética aditiva; $\mathrm{QM}_{\mathrm{M}}, \mathrm{QM}_{\mathrm{P}}$ e $\mathrm{Q} \mathrm{M}_{\mathrm{F} / \mathrm{M}}$ são os quadrados médios dos efeitos de macho, de progênie e de fêmea hierarquizada ao macho, respectivamente;

4) seleção baseada no índice combinado, em que a estratégia de seleção combinada foi realizada com a substituição dos valores das características do experimento pelo escore do índice combinado, para cada uma das progênies avaliadas, tendo-se considerado, simultaneamente, o desempenho individual de uma fêmea e o desempenho da família do macho na qual ela estava inserida. $\mathrm{O}$ índice combinado foi encontrado por meio do estimador: $\mathrm{IC}=\mathrm{h}_{\mathrm{M}}^{2}\left(\overline{\mathrm{Y}}_{\mathrm{m} . .}-\overline{\mathrm{Y}}_{\ldots}\right)+\mathrm{h}_{\mathrm{F} / \mathrm{M}}^{2}\left(\overline{\mathrm{Y}}_{\mathrm{mf} .}-\overline{\mathrm{Y}}_{\mathrm{m} . .}\right)$, em que: $\bar{Y}_{\text {... }}$ é a média geral; $\bar{Y}_{m}$. é a média do m-ésimo macho; e $\bar{Y}_{\mathrm{mf}}$ é a média da f-ésima fêmea acasalada com o m-ésimo macho.

A predição dos ganhos foi realizada para chegar a um ideótipo em que se procurou obter famílias com o maior número possível de frutos por planta, sem que ocorresse diminuição nas médias das outras características, a não ser para espessura de casca e número de dias até a antese. Para todos os cálculos de ganhos, foi utilizado o programa Genes (Cruz, 2001), e o estimador escolhido foi baseado no diferencial de seleção.
Para o cálculo dos ganhos preditos, dentro de cada uma das alternativas de seleção, foram empregados os índices de seleção: Mulamba \& Mock (1978), Pesek \& Baker (1969) e Smith (1936) e Hazel (1943), além da seleção direta em número de frutos por planta (Cruz et al., 2004). Para cada um dos índices de seleção, foram utilizados pesos obtidos aleatoriamente, até que fosse encontrado o que previsse o maior ganho genotípico.

\section{Resultados e Discussão}

As análises de variância conjunta das características (Tabela 1) demonstraram a existência de variabilidade na população, com diferenças significativas pelo teste $\mathrm{F}$ $(\mathrm{p}<0,01)$ com relação às progênies. Os graus de liberdade relativos a progênies foram desdobrados em efeitos de machos e efeitos de fêmeas hierarquizadas a machos, conforme Delineamento I de Comstock \& Robinson (1948), o que demonstra haver diferenças significativas entre progênies para estes efeitos, com 1 ou $5 \%$ de probabilidade pelo teste $\mathrm{F}$.

A falta de significância das interações machos $\mathrm{x}$ ambientes e fêmeas dentro de machos e ambientes, para as características peso, comprimento e largura de frutos, e da interação machos $\mathrm{x}$ ambientes, para as características número de frutos por planta e número de dias até a antese, indica comportamento similar dessas características nos dois ambientes estudados. Isto possibilita a recomendação simultânea de genótipos superiores a serem avaliados em ambos os ambientes.

As estimativas dos coeficientes de herdabilidade no sentido restrito, para as médias das unidades de seleção entre famílias de machos, famílias de fêmeas e famílias de fêmeas hierarquizadas a machos estão apresentadas na Tabela 1. De acordo com Hanson (1963), a herdabilidade no sentido restrito refere-se à fração das diferenças fenotípicas entre os pais que se espera recuperar entre os descendentes. Assim, esta foi utilizada neste trabalho para os cálculos dos ganhos de seleção, pois a herdabilidade no sentido amplo considera a variabilidade genética total, que é transmitida apenas em parte aos descendentes.

Em geral, os valores dos coeficientes de herdabilidade mais elevados foram obtidos para famílias de machos, e os menores para famílias de fêmeas hierarquizadas a machos. Deve ser destacado que estimativas de valores altos de herdabilidade podem ocorrer para caracteres de pequena variância genética aditiva, desde que seja pequena a influência ambiental no caráter. 
Viana et al. (2004), com o objetivo de iniciar um programa de melhoramento para o norte e noroeste fluminenses, estimaram coeficientes de herdabilidade em uma população de maracujá-amarelo, para várias características, e encontraram para a análise conjunta dos ambientes de Campos dos Goytacazes e Macaé, alta herdabilidade no sentido amplo para a característica número de frutos $(92,10 \%)$ e para comprimento de frutos $(82,84 \%)$, o que indica situação favorável ao melhoramento dessas características. As características acidez e porcentagem de suco apresentaram as mais baixas herdabilidades, 19,07 e 0\%, respectivamente.

$\mathrm{Na}$ Tabela 2, estão apresentadas as estimativas dos ganhos preditos por meio da seleção direta e do uso de índices de seleção, para as alternativas de seleção testadas pelo Delineamento I. Em todos os índices de seleção, a alternativa que apresentou os menores ganhos preditos foi a seleção entre famílias de fêmeas hierarquizadas a machos, que foi inferior às demais alternativas. Nesta alternativa de seleção, todos os machos genitores que doaram grãos de pólen para as progênies terão seus descendentes representados entre os indivíduos selecionados. Assim, o fato de essa alternativa possuir a menor estimativa de ganho pode ser explicado pela alta influência dos genitores masculinos na descendência $(p<0,05)$, com forte agrupamento dentro de alguns machos, quando a seleção foi realizada sem estratificação dentro deste.
Essa influência dos genitores masculinos pode também ser notada, quando se comparam os ganhos obtidos na alternativa de seleção entre famílias de machos, que foi a segunda melhor alternativa na predição de ganhos, tendo sido menor apenas que a seleção combinada. Ressalta-se que as melhores progênies estão reunidas em um grupo de poucos genitores masculinos.

Ao se analisarem as alternativas de seleção, observou-se que a utilização do índice combinado foi a alternativa que apresentou os maiores ganhos preditos, nos diversos índices de seleção testados. Esses resultados concordam com Falconer (1987), que afirma que a seleção baseada nos índices combinados é tão boa ou superior aos outros métodos. Essa alternativa apresentou, para seleção direta em número de frutos por planta, ganho de $18,55 \%$, e os ganhos com esta alternativa foram $10,79,35,22$ e $254,23 \%$ maiores que os ganhos esperados para famílias de machos, famílias de fêmeas e famílias de fêmeas hierarquizadas a machos, respectivamente. A seleção combinada constitui alternativa em que se identificam genótipos superiores não só a partir de informações do indivíduo, mas também de sua família.

Furtado (1996) utilizou uma população de milho, estruturada no Delineamento I, e concluiu que a seleção combinada e a seleção entre famílias de fêmeas, por meio de índices de seleção, foram as melhores alternativas para o melhoramento daquela população.

Tabela 1. Análises de variância de características produtivas em maracujá-amarelo, e estimativas dos coeficientes de herdabilidade utilizados na predição dos ganhos genéticos, para a análise conjunta dos ambientes de Viçosa, MG e Miracema, RJ.

\begin{tabular}{|c|c|c|c|c|c|c|c|}
\hline \multirow{2}{*}{$\begin{array}{l}\text { Fonte de } \\
\text { variação }\end{array}$} & \multirow{2}{*}{$\begin{array}{l}\text { Graus de } \\
\text { liberdade }\end{array}$} & \multicolumn{6}{|c|}{ Quadrados médios ${ }^{(1)}$} \\
\hline & & $\mathrm{NF}$ & $\mathrm{PF}$ & $\mathrm{CF}$ & LF & $\mathrm{EC}$ & $\mathrm{DA}$ \\
\hline Ambiente (E) & 1 & $16.156,79^{*}$ & $141.274,09 * *$ & $0,14^{\mathrm{ns}}$ & $1.162,31 * *$ & $698,80^{*}$ & $90.655,57^{* *}$ \\
\hline Agrupamento (A) & 2 & $6.548,10$ & $20.174,35$ & 183,67 & 646,10 & 114,58 & $5.946,85$ \\
\hline $\mathrm{E} \times \mathrm{A}$ & 2 & 380,95 & 564,22 & 34,58 & 11,01 & 10,15 & 804,35 \\
\hline Repetição (R)/ExA & 12 & 307,69 & $2.454,82$ & 88,15 & 63,04 & 5,41 & 369,40 \\
\hline Progênies & 110 & $504,13 * *$ & $2.330,71 * *$ & $139,51 * *$ & $47,53 * *$ & $3,26 * *$ & $408,09 * *$ \\
\hline $\operatorname{Macho}(\mathrm{M}) / \mathrm{S}$ & 20 & $851,08^{*}$ & $5.958,12 * *$ & $362,46 * *$ & $113,18^{* *}$ & $6,20 * *$ & $830,44 * *$ \\
\hline Fêmea $(F) / M / S$ & 90 & $427,03 * *$ & $1.524,62 * *$ & $89,96 * *$ & $32,94 * *$ & $2,60 * *$ & $314,24 * *$ \\
\hline $\mathrm{E} \times \mathrm{M} / \mathrm{S}$ & 20 & $316,98^{\mathrm{ns}}$ & $969,67^{\mathrm{ns}}$ & $43,75^{\mathrm{ns}}$ & $19,92^{\mathrm{ns}}$ & $1,87 *$ & $279,52^{\mathrm{ns}}$ \\
\hline $\mathrm{E} \times \mathrm{F} / \mathrm{M} / \mathrm{S}$ & 90 & $247,47 * *$ & $588,05^{\mathrm{ns}}$ & $27,87^{\mathrm{ns}}$ & $15,58^{\mathrm{ns}}$ & $1,07 *$ & $207,68 * *$ \\
\hline Resíduo & 429 & 163,26 & 649,63 & 22,32 & 13,34 & 0,81 & 140,06 \\
\hline CV (\%) & - & 47,13 & 13,75 & 5,48 & 4,85 & 13,98 & 9,33 \\
\hline Média & - & 27,11 & 185,42 & 86,25 & 75,37 & 6,45 & 126,84 \\
\hline $\mathrm{h}_{\mathrm{M}}^{2}(2)$ & - & 59,97 & 89,56 & 90,49 & 85,33 & 69,83 & 74,82 \\
\hline $\mathrm{h}_{\mathrm{F}}^{2^{(2)}}$ & - & 33,65 & 76,11 & 78,15 & 67,54 & 44,19 & 50,61 \\
\hline $\mathrm{h}_{\mathrm{F} / \mathrm{M}}^{2}{ }^{(2)}$ & - & 20,22 & 59,21 & 61,67 & 49,59 & 28,14 & 33,45 \\
\hline
\end{tabular}

(1)NF, número de frutos por planta; PF, peso de frutos, em g; CF, comprimento de frutos, em mm; LF, largura de frutos, em mm; EC, espessura de casca, em mm; DA, número de dias até a antese. ${ }^{(2)} \mathrm{h}_{\mathrm{M}}^{2}, \mathrm{~h}_{\mathrm{F}}^{2} \mathrm{e} \mathrm{h}_{\mathrm{F} / \mathrm{M}}^{2}$ : coeficientes de herdabilidade no sentido restrito de média de macho, de fêmea

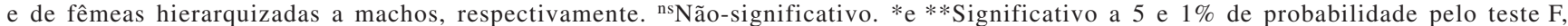
respectivamente. 
Ao selecionarem-se genótipos superiores para uma dada característica, mudanças indesejáveis podem ocorrer em outras, em razão da existência de correlações entre elas. Cruz et al. (2004) relataram que a seleção com base em uma única característica mostra-se inadequada, pois conduz a um produto final superior com relação à esta característica, mas pode levar a desempenhos não tão favoráveis para as demais. Uma forma de se aumentar o êxito com a seleção é por meio da seleção simultânea de características, assim, a utilização dos índices de seleção é alternativa eficiente, pois permitem a seleção com base em várias características de interesse.

Na utilização dos índices de seleção, foram testados pesos aleatórios até que se chegasse a um patamar de ganhos preditos como satisfatórios, ou seja, mais perto possível do ganho direto em número de frutos por

Tabela 2. Estimativas de ganhos preditos para maracujá-amarelo, em porcentagem, para as alternativas de seleção avaliadas, por meio de seleção direta na característica número de frutos por planta, e índices de seleção para a análise conjunta dos ambientes de Viçosa, MG e Miracema, RJ.

\begin{tabular}{|c|c|c|c|c|c|}
\hline \multirow{2}{*}{$\begin{array}{l}\text { Alternativas } \\
\text { de seleção }\end{array}$} & \multirow[t]{2}{*}{ Caracteristica $^{(1)}$} & \multicolumn{4}{|c|}{ Índices de seleção ${ }^{(2)}$} \\
\hline & & SD & $\mathrm{MM}$ & $\mathrm{PB}$ & $\mathrm{SH}$ \\
\hline \multirow[t]{6}{*}{ Entre machos } & NF & 16,75 & 12,10 & 12,50 & 10,08 \\
\hline & $\mathrm{PF}$ & $-4,75$ & 1,21 & 1,36 & $-0,29$ \\
\hline & $\mathrm{CF}$ & $-1,24$ & 0,07 & 1,07 & $-0,64$ \\
\hline & $\mathrm{LF}$ & $-1,23$ & 1,05 & 0,63 & $-0,13$ \\
\hline & $\mathrm{EC}$ & $-2,02$ & 3,88 & 1,71 & 4,34 \\
\hline & DA & $-3,48$ & $-1,81$ & $-1,95$ & $-3,72$ \\
\hline \multirow[t]{6}{*}{ Entre fêmeas } & NF & 13,72 & 12,10 & 13,73 & 7,65 \\
\hline & $\mathrm{PF}$ & $-4,11$ & 1,72 & 0,24 & 0,53 \\
\hline & $\mathrm{CF}$ & $-1,84$ & 0,89 & 0,06 & 0,29 \\
\hline & $\mathrm{LF}$ & $-0,73$ & 0,88 & 0,59 & $-0,22$ \\
\hline & $\mathrm{EC}$ & $-0,62$ & 0,31 & 0,75 & 3,47 \\
\hline & DA & $-2,15$ & $-1,50$ & $-1,71$ & $-2,82$ \\
\hline \multirow[t]{6}{*}{ Entre fêmeas/macho } & NF & 5,24 & 2,95 & 3,02 & 2,90 \\
\hline & $\mathrm{PF}$ & $-1,07$ & 2,02 & 2,06 & 1,94 \\
\hline & $\mathrm{CF}$ & $-0,32$ & 1,15 & 1,17 & 1,04 \\
\hline & LF & $-0,16$ & 0,73 & 0,84 & 0,26 \\
\hline & $\mathrm{EC}$ & 0,13 & 0,00 & 0,06 & 0,02 \\
\hline & DA & $-0,41$ & $-0,01$ & $-0,04$ & $-0,07$ \\
\hline \multirow[t]{6}{*}{ Seleção combinada } & NF & 18,55 & 15,85 & 15,92 & 11,17 \\
\hline & PF & $-5,80$ & 0,82 & 0,05 & 0,02 \\
\hline & $\mathrm{CF}$ & $-1,79$ & 0,35 & 0,17 & $-0,07$ \\
\hline & $\mathrm{LF}$ & $-2,08$ & 0,77 & 0,48 & $-0,11$ \\
\hline & $\mathrm{EC}$ & $-1,55$ & 1,55 & 0,92 & 3,70 \\
\hline & DA & $-3,21$ & $-2,80$ & $-2,75$ & $-1,75$ \\
\hline
\end{tabular}

${ }^{(1)} \mathrm{NF}$, número de frutos por planta; PF, peso de frutos, em g; $\mathrm{CF}$, comprimento de frutos, em $\mathrm{mm}$; LF, largura de frutos, em mm; EC, espessura de casca, em mm; DA, número de dias até a antese. ${ }^{(2)} \mathrm{SD}$, seleção direta em número de frutos por planta; MM, Mulamba \& Mock (1978); PB, Pesek \& Baker (1969); SH, Smith (1936) e Hazel (1943). planta, sem que ocorresse queda em outras características. Cruz (1990) considerou como peso mais apropriado o uso do coeficiente de variação genético na predição de ganhos em milho. Martins (1999) e Granate et al. (2002), ao trabalhar com eucalipto e milho-pipoca, respectivamente, não obtiveram resultados desejados ao utilizá-los na predição de ganhos. Neste trabalho, o uso do coeficiente de variação foi testado como peso para os índices, mas mostraram-se inadequados, pois, apresentaram ganhos pequenos e, para algumas características, negativos.

O índice de Smith (1936) e Hazel (1943) foi o que apresentou o menor ganho predito nas diversas alternativas, tendo resultado em ganhos insatisfatórios, quando comparado aos outros índices testados.

Os outros índices testados, Mulamba \& Mock (1978) e Pesek \& Baker (1969), apresentaram ganhos semelhantes, foram eficazes na escolha das melhores progênies para recombinação e tiveram seus maiores ganhos na seleção combinada. O índice de Mulamba \& Mock (1978) foi de: 15,85\% para número de frutos por planta (NF); 0,82\% para peso de frutos (PF); 0,35\% para comprimento de frutos $(\mathrm{CF}) ; 0,77 \%$ para largura de frutos (LF); 1,55\% para espessura de casca (EC); e de -2,80\% para número de dias até a antese (DA). O índice de Pesek \& Baker (1969) apresentou ganhos de 15,92\% para NF; $0,05 \%$ para $\mathrm{PF} ; 0,17 \%$ para $\mathrm{CF} ; 0,48 \%$ para LF; $0,92 \%$ para EC; e $-2,75 \%$ para DA.

Dessa forma, optou-se pelas 35 progênies selecionadas pelo índice de Mulamba \& Mock (1978), por meio do uso do índice combinado, já que apresentou ganhos ligeiramente superiores aos de Pesek \& Baker (1969), exceto quanto à característica número de frutos por planta.

\section{Conclusões}

1. O uso de índices de seleção permite a predição de ganhos simultâneos em várias características do maracujá-amarelo, o que não é possível com a seleção direta.

2. A seleção combinada é a alternativa mais indicada de seleção, com os maiores ganhos preditos para a característica número de frutos por planta.

3. Os índices de Mulamba \& Mock e Pesek \& Baker são os mais adequados, com maiores ganhos preditos, quando comparados aos de Smith e Hazel. 


\section{Referências}

COMSTOCK, R.E.; ROBINSON, H.F. The components of genetic variance in populations of biparental progenies and their use in estimating the average degree of dominance. Biometrics, v.4, p.254$266,1948$.

CRUZ, C.D. Aplicação de algumas técnicas multivariadas no melhoramento de plantas. 1990. 188p. Tese (Doutorado) - Escola Superior de Agricultura Luiz de Queiroz, Piracicaba.

CRUZ, C.D. Programa Genes (versão Windows): aplicativo computacional em genética e estatística. Viçosa: UFV, 2001. 648p.

CRUZ, C.D.; CARNEIRO, P.C.S. Modelos biométricos aplicados ao melhoramento genético. Viçosa: UFV, 2003. v.2. 585p.

CRUZ, C.D.; REGAZZI, A.J.; CARNEIRO, P.C.S. Modelos biométricos aplicados ao melhoramento genético. 3.ed. Viçosa: UFV, 2004. v.1. 480p.

CRUZ, C.D.; VENCOVSKY, R.; SILVA, S.O. e; TOSELLO, G.A. Comparison of gains from selection among corn progenies, based on different criteria. Revista Brasileira de Genética, v.16, p.79-89, 1993.

ELSTON, R.C. A weight-free index for the purpose of ranking on selection with respect to several traits at a time. Biometrics, v.19, p.85-97, 1963.

EYHERABIDE, G.H.; HALLAUER, A.R. Reciprocal full-sib recurrent selection in maize: direct and indirect responses. Crop Science, v.31, p.952-959, 1991.

FALCONER, D.S. Introdução à genética quantitativa. Viçosa: UFV, 1987. 279p.

FURTADO, M.R. Alternativas de seleção no Delineamento I de Comstock e Robinson, em milho. 1996. 94p.Tese (Doutorado) Universidade Federal de Viçosa, Viçosa.
GRANATE, M.J.; CRUZ, C.D.; PACHECO, C.A.P. Predição de ganho genético com diferentes índices de seleção no milho-pipoca CMS-43. Pesquisa Agropecuária Brasileira, v.37, p.1001-1008, 2002.

HALLAUER, A.R.; MIRANDA FILHO, J.B. Quantitative genetics in maize breeding. $2^{\text {nd }}$ ed. Ames: Iowa State University Press, 1988. 468p.

HANSON, W.D. Heritability. In: HANSON, W.D.; ROBINSON, H.F. (Ed.). Statistical genetics and plant breeding. Washington: National Academy of Science; National Research Council, 1963. p.125-139.

HAZEL, L.N. The genetic basis for constructing selection indexes. Genetics, v.28, p.476-490, 1943.

MARTINS, I.S. Comparação entre métodos uni e multivariados aplicados na seleção em Eucalyptus grandis. 1999. 94p. Tese (Doutorado) - Universidade Federal de Viçosa, Viçosa.

MULAMBA, N.N.; MOCK, J.J. Improvement of yield potential of the Eto Blanco maize (Zea mays L.) population by breeding for plant traits. Egyptian Journal of Genetics and Cytology, v.7, p.40-57, 1978.

PEREIRA, M.G.; AMARAL JÚNIOR, A.T. do. Estimation of genetic components in popcorn based on the nested design. Crop Breeding and Applied Biotechnology, v.1, p.3-10, 2001.

PESEK, J.; BAKER, R.J. Desired improvement in relation to selected indices. Canadian Journal of Plant Sciences, v.49, p.803-804, 1969.

SMITH, H.F. A discriminant function for plant selection. Annual Eugenics, v.7, p.240-250, 1936.

VIANA, A.P.; PEREIRA, T.N.S.; PEREIRA, M.G.; AMARAL JÚNIOR, A.T. do; SOUZA, M.M. de; MALDONADO, J.F.M. Parâmetros genéticos em populações de maracujazeiro-amarelo. Revista Ceres, v.51, p.545-555, 2004.

WILLIAMS, J.S. The evaluation of a selection index. Biometrics, v.18, p.375-393, 1962.

Recebido em 23 de maio de 2006 e aprovado em 27 de outubro de 2006 